\title{
Calibration and validation of individual GOCE accelerometers by precise orbit determination
}

\author{
P. N. A. M. Visser $^{1}$ • J. A. A. van den IJssel ${ }^{1}$
}

Received: 8 July 2015 / Accepted: 26 August 2015 / Published online: 14 September 2015

(C) The Author(s) 2015. This article is published with open access at Springerlink.com

\begin{abstract}
The European Space Agency Gravity field and steady-state Ocean Circular Explorer (GOCE) carries a gradiometer consisting of three pairs of accelerometers in an orthogonal triad. Precise GOCE science orbit solutions (PSO), which are based on satellite-to-satellite tracking observations by the Global Positioning System and which are claimed to be at the few $\mathrm{cm}$ precision level, can be used to calibrate and validate the observations taken by the accelerometers. This has been done for each individual accelerometer by a dynamic orbit fit of the time series of position co-ordinates from the PSOs, where the accelerometer observations represent the non-gravitational accelerations. Since the accelerometers do not coincide with the center of mass of the GOCE satellite, the observations have to be corrected for rotational and gravity gradient terms. This is not required when using the so-called common-mode accelerometer observations, provided the center of the gradiometer coincides with the GOCE center of mass. Dynamic orbit fits based on these common-mode accelerations therefore served as reference. It is shown that for all individual accelerometers, similar dynamic orbit fits can be obtained provided the abovementioned corrections are made. In addition, accelerometer bias estimates are obtained that are consistent with offsets in the gravity gradients that are derived from the GOCE gradiometer observations.
\end{abstract}

Keywords GOCE - Accelerometer - Calibration . Validation - Precise orbit determination - Bias - Drift . Gravity gradients

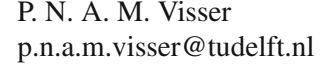

P. N. A. M. Visser

p.n.a.m.visser@tudelft.nl

1 Faculty of Aerospace Engineering, Delft University of Technology, Kluyverweg 1, 2629 HS Delft, The Netherlands

\section{Introduction}

The Gravity field and steady-state Ocean Circulation Explorer (GOCE) is the first European Space Agency (ESA) earth explorer, launched on March 11, 2009. More than 4 years of successful mission operations ended on November 11, 2013, when GOCE re-entered the atmosphere. Its primary mission objective was to map the Earth's mean gravity field with a precision of $1 \mathrm{mgal}$ and $1 \mathrm{~cm}$ in terms of gravity anomalies and geoid heights, respectively, for spatial scales better than $100 \mathrm{~km}$ (Drinkwater et al. 2007). To this aim, GOCE was equipped with a gradiometer, consisting of an orthogonal triad of three pairs of accelerometers, a high-precision dual-frequency Global Positioning System (GPS) receiver, star trackers, and ion engines as part of a drag-free control (DFC) system. In order to meet its mission objectives, the GOCE instruments needed to be precisely calibrated. Several procedures and methods were defined and used to calibrate the satellite gravity gradient (SGG) observations (Rispens and Bouman 2009; Siemes et al. 2012). These SGG observations are derived from the observations collected by the gradiometer's six accelerometers. These procedures proved successful and it has been shown that the SGG observations are scaled with a precision better than $10^{-3}$ (Bouman et al. 2011). Since the gradiometer is designed to work in a bandwidth of $5-100 \mathrm{mHz}$, it is especially the scale that needs to be precisely known. The calibrated SGG observations, therefore, still include (drifting) offsets or biases.

In addition to SGG observations, the so-called commonmode accelerations can be derived by averaging for each gradiometer arm or axis the observations of the associated pair of accelerometers. These common-mode accelerations represent the non-gravitational accelerations that are not compensated —or induced — by the DFC, provided the gradiometer's center coincides with the satellite's center of mass. 
The latter is the case to within a few $\mathrm{cm}$. The common-mode accelerations are used to support long-wavelength gravity field information from the GOCE GPS observations. However, also these common-mode accelerations are biased and the associated biases need to be estimated at regular time intervals (Pail et al. 2011). These common-mode biases in fact represent the average of biases for the associated pairs of individual accelerometers. It is interesting to investigate if the biases can be determined for the individual accelerometers as well. It has to be realized, however, that the location of the individual accelerometers does not coincide with the GOCE center of mass. The offset is between 25.0 and $25.7 \mathrm{~cm}$, or half the length of the gradiometer arms.

This paper will show that the GOCE individual accelerometers can be calibrated and validated by precise orbit determination (POD). It will thus also be shown that such a calibration can be done for accelerometers that are not located in the center of mass of a satellite. A methodology was adopted that was designed and implemented before launch of the GOCE satellite. This method was tested by an endto-end simulation and is described in detail in (Visser 2009). A multi-year dataset of real GOCE observations was used to further assess the capabilities of this methodology. This dataset is described in Sect. 2. The adopted methodology is summarized in Sect. 3, followed by an overview of obtained results (Sect. 4) and conclusions (Sect. 5).

\section{Observations}

As outlined in (Visser 2009), the GOCE orbit and accelerometer calibration parameters can be determined from the GPS Satellite-to-Satellite Tracking (SST) observations. For the method outlined in this paper, use is made of the pre- cise science orbit (PSO) solutions produced by the GOCE high-level processing facility, i.e.,the SST observations are not used directly, but have already been reduced to time series of-in this case-kinematic GOCE orbit co-ordinates (Bock et al. 2011). These co-ordinates serve as the observables and are provided as Cartesian $x, y$, and $z$ co-ordinates in an Earth-centered, Earth-fixed (ECF) co-ordinate frame. They are available together with rotation parameters that establish the connection between the ECF and J2000 earthcentered pseudo-inertial reference frame (Montenbruck and Gill 2000). The claimed precision of the PSO product is around $2 \mathrm{~cm} 1$ dimensionally (1D) (Bock et al. 2011).

The accelerometer observations are provided as time series in the gradiometer reference frame (GRF). The orientation of this GRF in the J2000 reference frame is provided by quaternions derived from star tracker observations. The results described in this paper (Sect. 4) are based on GOCE data covering the period November 1, 2009-October 20, 2013, i.e., the full operational period.

The six accelerometers of the gradiometer are schematically displayed in Fig. 1 (largely taken from Visser 2009). The locations of the accelerometers are indicated along the $X, Y$, and $Z$ axes of the GRF. These axes are predominantly aligned with the along-track (or flight), cross-track, and radial (or height) directions, respectively. Each accelerometer has two ultra-precise axes and one less precise axis. It will be shown in the remainder of this paper that the POD confirms the difference in sensitivity of the different accelerometer axes.

\section{Methodology}

As outlined in (Visser 2009), it is important to correct the observations of the individual accelerometers for rotational

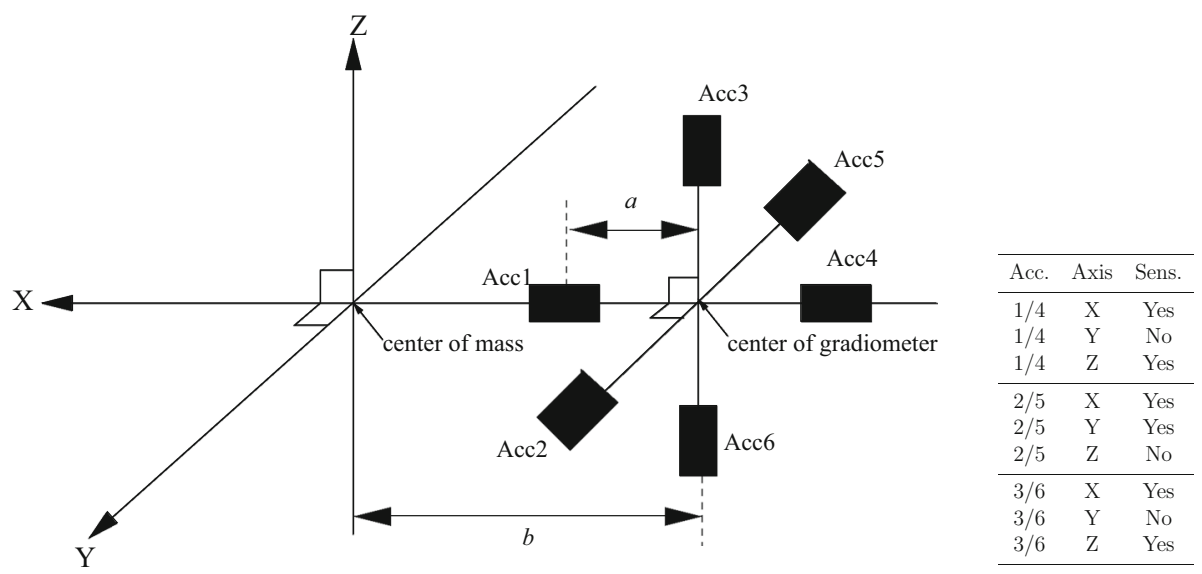

Fig. 1 Configuration and naming convention of the three orthogonal pairs of accelerometers that form together the GOCE gravity gradiometer. The offset of the accelerometers is indicated by $a$, where $a$ is equal to half the arm length for the associated axis. This arm length is either equal to 50.0 or $51.4 \mathrm{~cm}$ (Cesare et al. 2005). The offset of the center of the gradiometer with respect to the center of mass of the satellite is indicated by $b$ (taken equal to zero for this study). The sensitive and less sensitive axes are indicated as well 
and gravity gradient terms due to their different center of mass offsets. All accelerometers in principle experience identical non-gravitational linear accelerations d. For this paper, the following representation is used [cf. Eqs. (7.2.1) and (7.2.2) in ESA 1999]:

$\mathbf{d}=\mathbf{S}_{i}^{-1}\left(\mathbf{a}_{\mathrm{obs}, i}-\mathbf{b}_{i}-\epsilon_{i}\right)-(\Gamma+\mathbf{R}) \mathbf{x}_{\mathbf{i}}$,

where $\mathbf{S}_{i}$ represents the $3 \times 3$ diagonal scale factor matrix, and $\mathbf{a}_{\mathrm{obs}, i}, \mathbf{b}_{i}, \epsilon_{i}$, respectively, the 3-dimensional vectors of observations, biases, and observation errors, all for accelerometer $i(i=1, \ldots, 6)$. The gravity gradient tensor and matrix with rotational terms are represented by $\Gamma$ and R. Finally, the offset of accelerometer $i$ with respect to the satellite's center of mass is indicated by $\mathbf{x}_{i}$, cf. (Visser 2008).

The (diagonal) matrix of accelerometer scale factors $S_{i, k}$ is represented by $\mathbf{S}_{i}$

$\mathbf{S}_{i}=\left(\begin{array}{ccc}S_{i, x} & 0 & 0 \\ 0 & S_{i, y} & 0 \\ 0 & 0 & S_{i, z}\end{array}\right)$,

where $k=(x, y, z)$ with $x, y$, and $z$ the axes of the GRF, which are very closely aligned with the axes of the accelerometer reference frames (for this study it is assumed that they are perfectly aligned). Given the high quality of the scale factor calibration for the GOCE gradiometer, they are taken equal to 1 (Bouman et al. 2011).

$\Gamma$ represents the gravity gradient matrix containing the second-order derivatives of the gravitational field potential $\Gamma_{k l}(k, l=x, y, z)$ at the satellite location:

$\Gamma=\left(\begin{array}{lll}\Gamma_{x x} & \Gamma_{x y} & \Gamma_{x z} \\ \Gamma_{y x} & \Gamma_{y y} & \Gamma_{y z} \\ \Gamma_{z x} & \Gamma_{z y} & \Gamma_{z z}\end{array}\right)$.

The matrix with rotational terms $\mathbf{R}$ is written as follows:

$$
\begin{aligned}
\mathbf{R}= & \left(\begin{array}{lll}
r_{x x} & r_{x y} & r_{x z} \\
r_{y x} & r_{y y} & r_{y z} \\
r_{z x} & r_{z y} & r_{z z}
\end{array}\right) \\
= & \left(\begin{array}{ccc}
-\omega_{y}{ }^{2}-\omega_{z}{ }^{2} & \omega_{y} \omega_{x} & \omega_{z} \omega_{x} \\
\omega_{x} \omega_{y} & -\omega_{x}^{2}-\omega_{z}^{2} & \omega_{z} \omega_{y} \\
\omega_{x} \omega_{z} & \omega_{y} \omega_{z} & -\omega_{x}{ }^{2}-\omega_{y}{ }^{2}
\end{array}\right) \\
& +\left(\begin{array}{ccc}
0 & -\dot{\omega}_{z} & \dot{\omega}_{y} \\
\dot{\omega}_{z} & 0 & -\dot{\omega}_{x} \\
-\dot{\omega}_{y} & \dot{\omega}_{x} & 0
\end{array}\right),
\end{aligned}
$$

where $\omega_{k}$ and $\dot{\omega}_{k}$ represent the angular rotation rates $\omega^{T}=$ $\left(\omega_{x}, \omega_{y}, \omega_{z}\right)$ and the angular acceleration rates $\dot{\omega}^{T}=$ $\left(\dot{\omega}_{x}, \dot{\omega}_{y}, \dot{\omega}_{z}\right)$.

The angular rotation rates and accelerations are obtained by taking the first and second time derivatives of the rotation angles $\phi_{i}$ around the GRF axes $i=x, y, z$ (also referred to as roll, pitch, and yaw angles). They are derived from the merged EGG_IAQ product by using a moving time window of certain width over the time series of the rotation angles and fitting second-order polynomials, cf. Eq. (5) in (Visser 2009):

$\phi_{i}=\phi_{0, i}+\omega_{i} t+\frac{1}{2} \dot{\omega}_{i} t^{2}$,

where $t$ represents time and $\phi_{0, i}$ the orientation angle at the start of the time interval. The EGG_IAQ product provides the orientation of the gradiometer in the J2000 reference frame based on a combination of star tracker observations (for low frequencies) and angular accelerations derived from the accelerometer observations (for the higher frequencies) (Frommknecht et al. 2011). The time interval of the EGG_IAQ product is $1 \mathrm{~s}$. An estimation window of $25 \mathrm{~s}$ was applied to estimate the rotational velocities and accelerations. This time interval was found to lead to the best consistency between individual and common-mode accelerometer observations in terms of root-mean-square (RMS) about mean: see e.g., Figs. 3, 4 and 5 in Sect. 4 for accelerometers 1, 2, and 3. It has to be noted that the patterns for accelerometers 4 , 5 , and 6 are predominantly mirror images of the patterns for the accelerometers 1, 2, and 3 displayed in these Figures. The nominal common-mode combination was always used, which means that for the $\mathrm{X}, \mathrm{Y}$, and $\mathrm{Z}$ axes, respectively, the average of the observations of accelerometers 1 and 4,2 and 5 , and 3 and 6 , was taken.

The NASA Goddard Space Flight Center GEODYN software was used for the orbit determination and estimation of accelerometer bias parameters (Pavlis et al. 2006). A dynamic orbit determination technique is used, where an iterative Bayesian least-squares method is used to solve the linearized observation equations. These observation equations are obtained by numerical integration of the equations of motion and the so-called variational equations (Montenbruck and Gill 2000). Use was made of 24-h arcs (midnight to midnight), where for each arc the GOCE initial position and velocity and for each accelerometer axis one bias was estimated. Thus, in total, nine parameters are estimated for each orbital arc. For each individual accelerometer, and for the common-mode, a separate orbit determination run is conducted. Furthermore, it is noted that a uniform weighting scheme was adopted, where all kinematic co-ordinates are weighted equally. It is realized that the PSO product includes covariance information for the estimated kinematic positions that can be used for a more optimal weighting scheme, but it will be shown below that the uniform weighting already leads to very consistent accelerometer bias and also scale factor estimates.

It is interesting to note that in the simulations described in (Visser 2009), several parameter estimation schemes were 
Table 1 Standards and models used for the GOCE orbit data reduction and estimation of accelerometer bias parameters

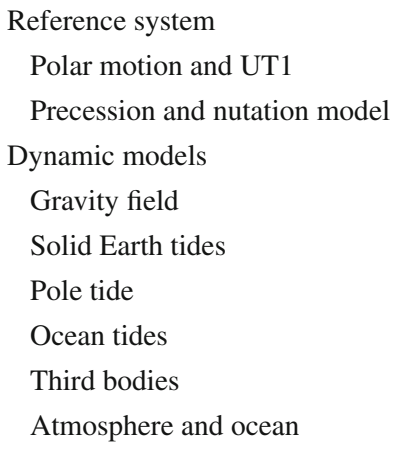

\author{
IERS EOP 05 C04 (IAU2000A) series with IERS 2003 daily and sub-daily corrections \\ IERS Conventions 2003 (McCarthy et al. 2004)
}

GOCO03s complete to degree and order 200 including secular degree 2 terms (GOCO 2014) IERS Conventions 2003 (McCarthy et al. 2004)

IERS Conventions 2003 (McCarthy et al. 2004)

GOT 4.7, update to Ray (1999)

Sun, Moon, Venus, Mars and Jupiter (DE-405) (Standish 1998)

GOCE de-aliasing product (Gruber et al. 2010) tested, including the estimation of empirical accelerations to absorb e.g.,gravity field model errors and also the estimation of gravity field coefficients themselves. Moreover, the estimability of accelerometer scale factors was assessed leading to the conclusion that no reliable estimates could be made for the $X$ axis due to the lack of signal left by the DFC (it will be shown in Sect. 4, however, that it is possible to estimate these scale factors because of occasional maneuvering). It was found that there is no need to estimate empirical accelerations because of the quality of recent gravity field models and other force models (Table 1).

An overview of the adopted standards and dynamic models is displayed in Table 1. The reference system used for the PODs is consistent with the IERS 2003 conventions (McCarthy et al. 2004). In addition, a comprehensive gravitational force modeling is adopted, including the GOCO03s gravity field model complete to degree and order 200, solid earth and ocean tides, third-body perturbations, and gravity field variations due to atmosphere and ocean mass changes (GOCO 2014; McCarthy et al. 2004; Ray 1999; Gruber et al. 2010). It has to be noted that the GOCO03s model was not only used in the orbit determination and estimation of accelerometer biases, but also for deriving the gravity gradient center of mass offset corrections for the individual accelerometers (Eq. 1). Moreover, the modeled gravity gradients served as a validation for the consistency between GOCE observed gravity gradients and estimated biases for the individual accelerometers (Sect. 4).

\section{Results}

GOCE is equipped with a DFC system that compensates the non-gravitational forces in the direction of the GRF $X$ axis (predominantly the flight direction) to below a level of just a few $\mathrm{nm} / \mathrm{s}^{2}$ in terms of acceleration. This DFC system does, however, not compensate the non-gravitational forces in directions perpendicular to this $X$ axis. Typically, the non-gravitational acceleration for the $Y$ (predominantly cross-track) and $Z$ (predominantly radial) axes is of the order of 100 to a few tens of $\mathrm{nm} / \mathrm{s}^{2}$. GOCE is flying in a Sunsynchronous orbit in order to maximize the required solar power for the ion engines, which results in a more or less persistent solar radiation pressure force predominantly in the $Y$ direction. It is interesting to note that occasionally the DFC is offset by an acceleration up to more than $1000 \mathrm{~nm} / \mathrm{s}^{2}$ for orbit correction maneuvers. The latter can be used for estimating reliable scale factors for the accelerometers especially for the $X$ axis of the accelerometers as well in addition to biases (Sect. 4.3).

\subsection{Center of mass offset corrections}

Figure 2 displays a typical 1-day time series of (biased) non-gravitational GOCE accelerations as taken from the common-mode accelerometer observations on November 15 , 2009. The daily RMS-about-mean for the common-mode accelerations is about 2,86 , and $28 \mathrm{~nm} / \mathrm{s}^{2}$ for the $X, Y$, and $Z$ axes, respectively. This shows the very good level of drag compensation in the predominantly flight direction for this day. It was found that the consistency between the variations of the fully corrected individual accelerometer observations with the common-mode accelerations is always two to three orders of magnitude below these values for the $Y$ and $Z$ axes, and one to two orders of magnitude for the $X$ axis (for which the signal is very small). This is shown for November 15, 2009 as well in Figs. 3, 4, and 5.

Before applying the center of mass offset corrections, the RMS-about-mean of differences between the observations of the individual accelerometers can be of the order of $40 \mathrm{~nm} / \mathrm{s}^{2}$, especially for the $Z$ axis (e.g., accelerometer 2 in Fig. 5). After applying the rotational corrections, this is reduced to less than $6 \mathrm{~nm} / \mathrm{s}^{2}$. Finally, after applying the gravity gradient corrections as well, the consistency is better than $1 \mathrm{~nm} / \mathrm{s}^{2}$ and even down to less than $0.02 \mathrm{~nm} / \mathrm{s}^{2}$ for some of the sensitive $X$ and $Z$ axes. It can be observed as well that some accelerometers display a small drift, of the order of $0.4 \mathrm{~nm} / \mathrm{s}^{2}$ per day (e.g., for the $Y$ axis of accelerometer 2 in Fig. 4). 

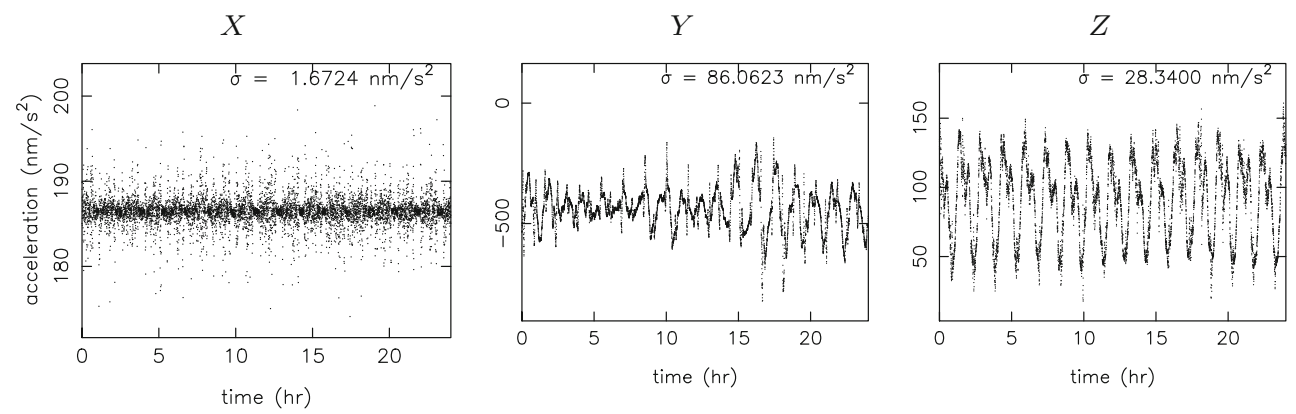

Fig. 2 Common-mode accelerations along the GRF $X, Y$, and $Z$ axes. The selected day is November 15, 2009. Please note that these accelerations have not been corrected for the common-mode accelerometer biases

$X$ axis

Accelerometer
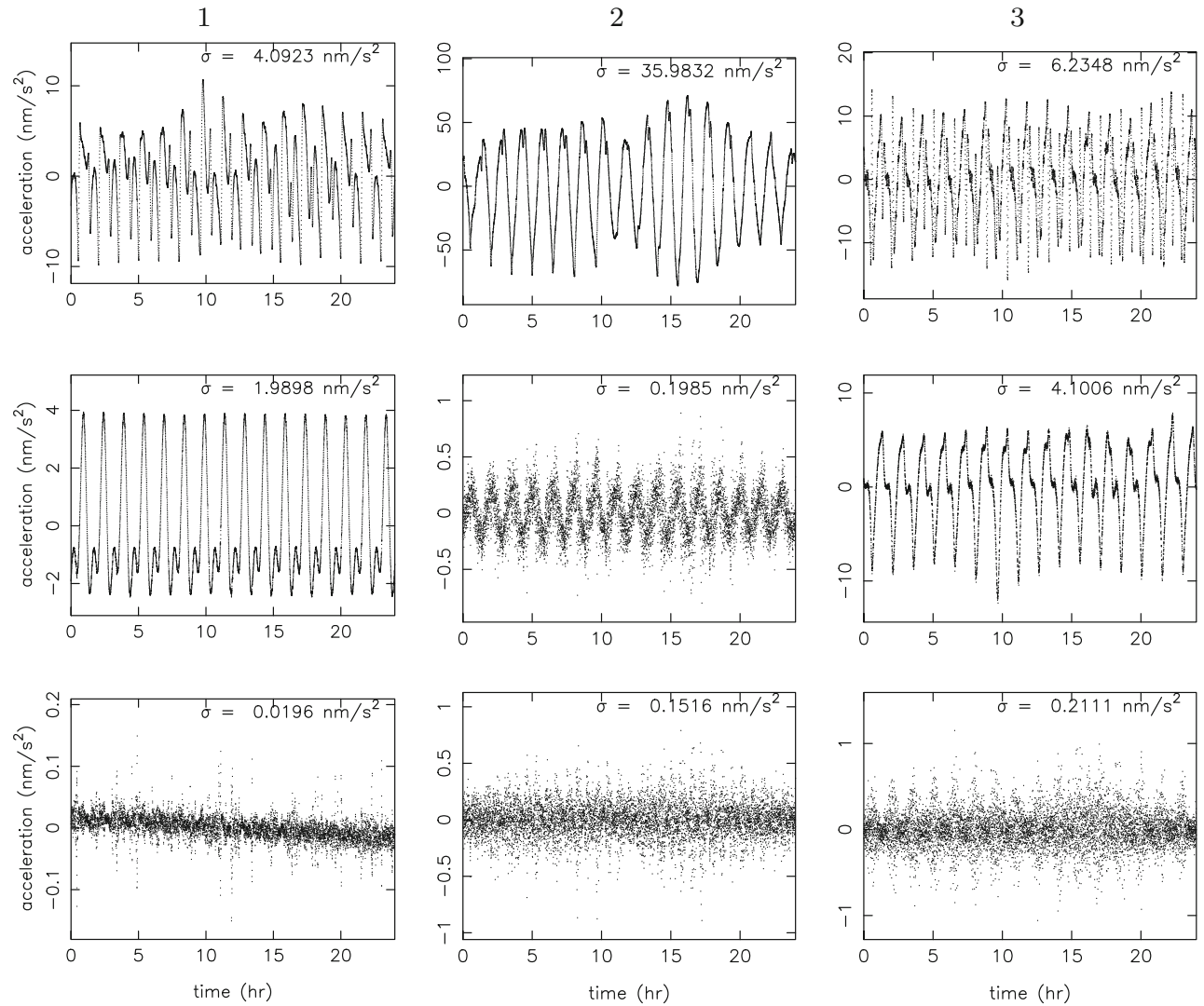

Fig. 3 Differences between common-mode accelerations and observations from accelerometers 1,2 , and 3 for the GRF $X$ axis. The differences are displayed before corrections (top row), after applying rotational cor-

rections (middle row), after applying both rotational and gravity gradient corrections (bottom row), and after taking away the mean. The selected day is November 15, 2009

As stated above, for each individual accelerometer, a separate orbit determination run is conducted for each day (from midnight to midnight), where in addition to the initial position and velocity, for each axis, a bias is estimated (nine parameters in total). As also stated above, some of the accelerometer axes display small drifts. These drifts are of the order of a few tenths of a $\mathrm{nm} / \mathrm{s}^{2}$ per day, which is significantly below the precision with which the daily biases can be estimated (see e.g., Fig. 6). Therefore, these drifts were not co-estimated directly with the daily biases, but were derived later on from the long time series of these daily biases.

The RMS-of-fit $(\mathrm{cm})$ of PSO kinematic co-ordinates for daily arcs obtained by the dynamic orbit computation is displayed in Table 2. It can be observed that for all accelerometers almost identical fits are obtained, ranging between 6.87 and $6.97 \mathrm{~cm}$ for the full GOCE operational mission 

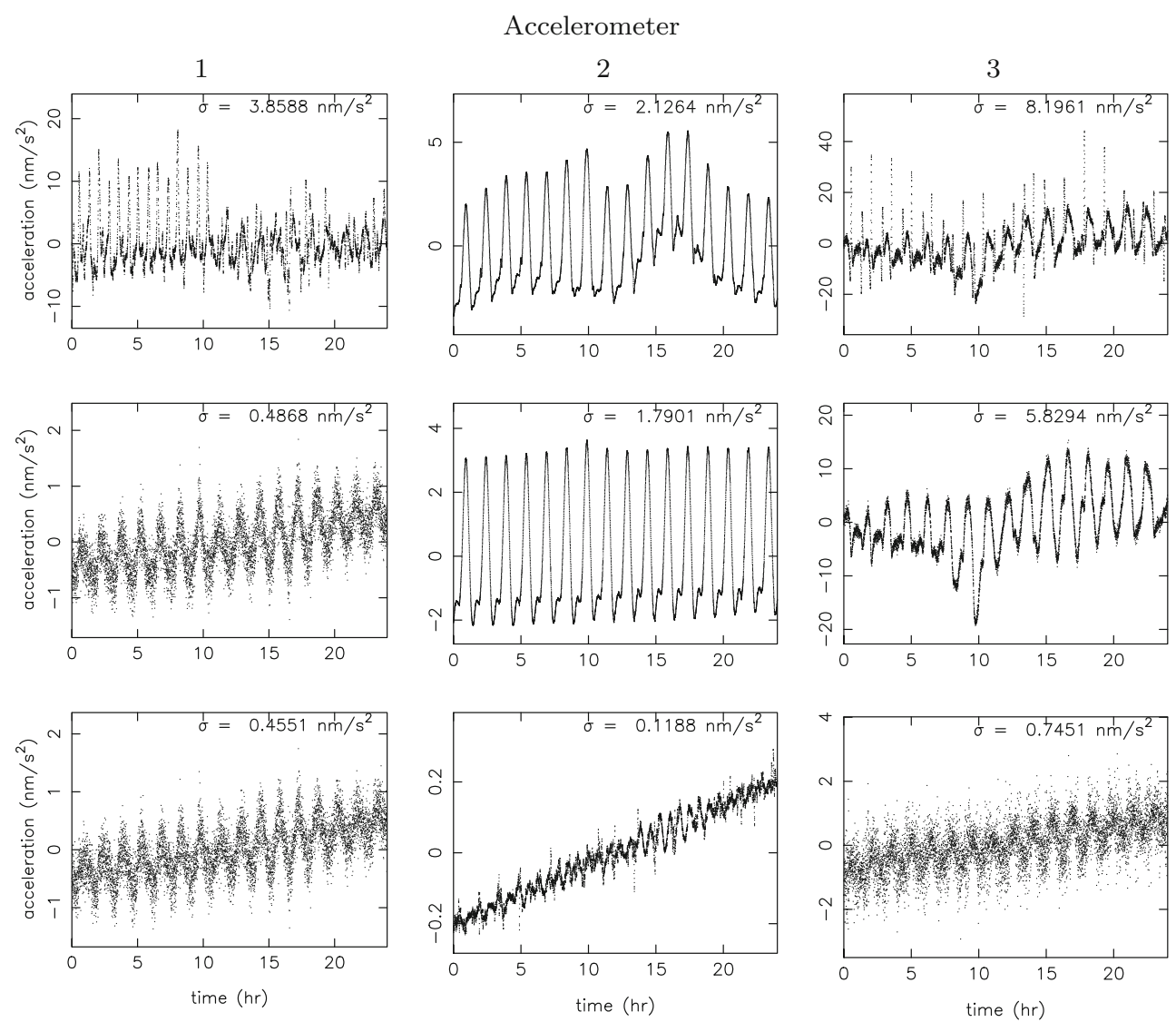

Fig. 4 Differences between common-mode accelerations and observations from accelerometers 1, 2, and 3 for the GRF $Y$ axis. The differences are displayed before corrections (top row), after applying rotational cor-

period. These fits are also almost identical to the fit of $6.87 \mathrm{~cm}$ obtained when using the common-mode accelerometer observations. A test period of 10 days (1-10 November 2009) was used to assess the impact of the rotational and gravity gradient accelerometer corrections on the RMS-offit. Without these corrections, the fit values can be as large as almost $15 \mathrm{~cm}$, and the values for the several individual accelerometers are not consistent. Based on the orbital fits, it can be concluded that the corrections are required and have been properly implemented. It is interesting to note that for the accelerometers 1 and 4, which are located on the GRF $X$ axis arm, already a fit comparable to the common-mode one is obtained without corrections. However, the corrections are required for these accelerometers as well in order to have correct estimates for their biases and to prevent that these biases include mean offsets due to the rotational terms and gravity gradients. The fit for $1-10$ November is around $5.2 \mathrm{~cm}$, which is below about $6.2 \mathrm{~cm}$ for the entire GOCE operational mission period. This can be explained by the slight degradation of the quality of the kinematic position co-ordinates, which is due to the growing ionospheric perturbations during the rections (middle row), after applying both rotational and gravity gradient corrections (bottom row), and after taking away the mean. The selected day is November 15, 2009

GOCE mission lifetime that affects the GPS receiver measurements (Bock et al. 2014).

\subsection{Accelerometer and gravity gradient biases}

In the period from November 1, 2009 to October 20, 2013, 1213 daily arcs could be identified without gaps in the GPS, accelerometer, and star tracker observations. The estimated accelerometer biases are displayed for these days in Figs. 6 and 7. These Figures include a least-squares linear fit of the daily biases as well allowing the estimation of drifts of the accelerometers. The less sensitive axes can be observed clearly given their much larger biases, up to more than $20,000 \mathrm{~nm} / \mathrm{s}^{2}$ and in some cases also much higher drifts, up to more than $3 \mathrm{~nm} / \mathrm{s}^{2} /$ day. For the sensitive axes, the biases and drifts are maximally around $300 \mathrm{~nm} / \mathrm{s}^{2}$ and $0.2 \mathrm{~nm} / \mathrm{s}^{2} / \mathrm{day}$, respectively. The best fits for the linear regression are obtained for the $X$ axis, typically much better than $1 \mathrm{~nm} / \mathrm{s}^{2}$. For the $Y$ axis, these fits are at the $10-30 \mathrm{~nm} / \mathrm{s}^{2}$ level, except for the less sensitive axes for which values up 
$Z$ axis

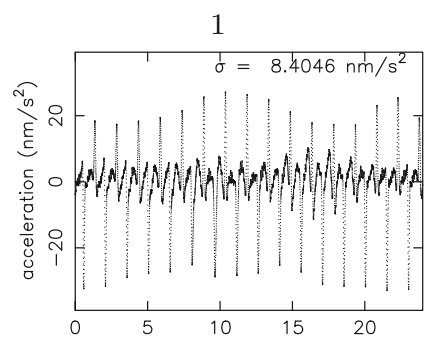

Accelerometer
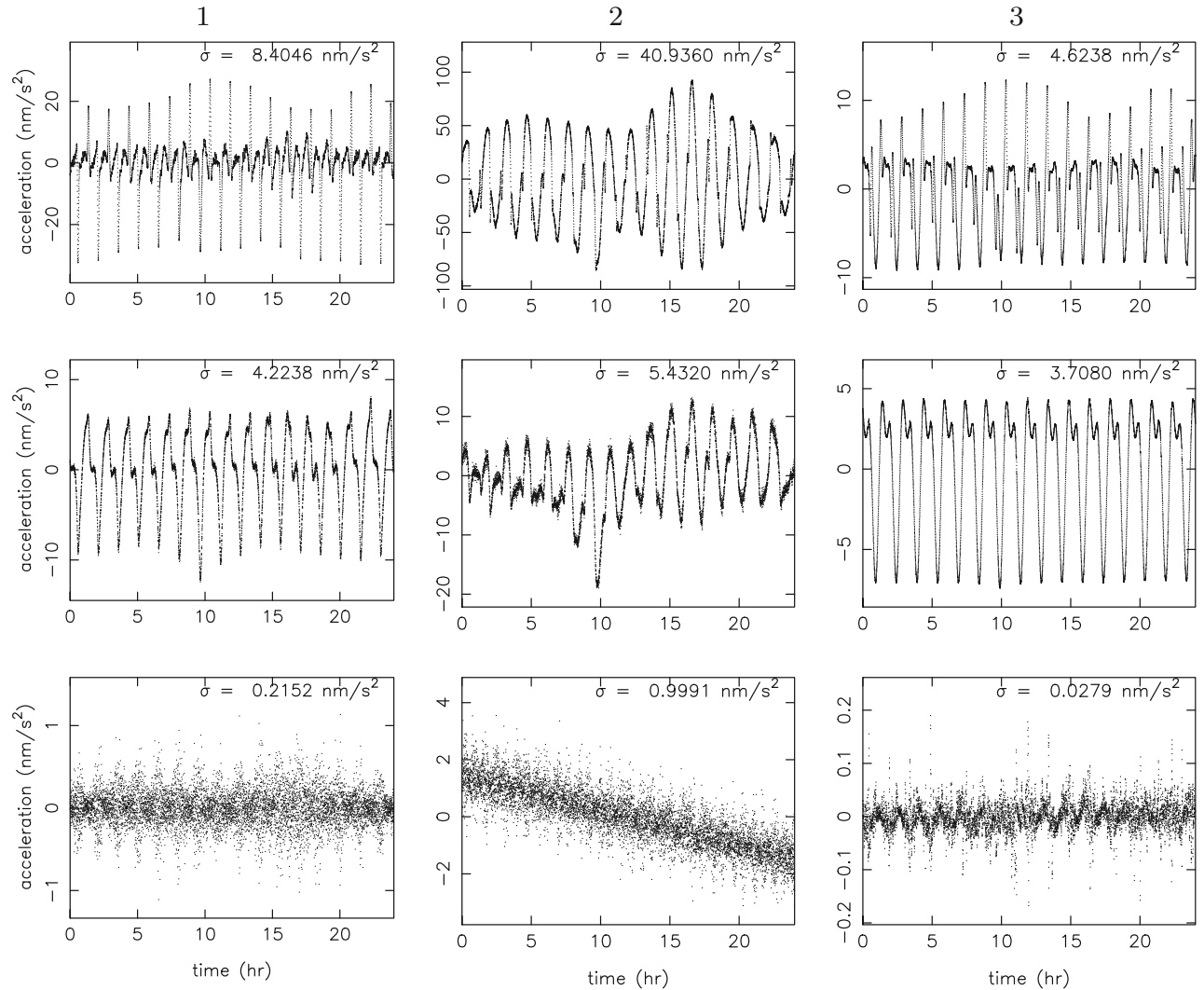

Fig. 5 Differences between common-mode accelerations and observations from accelerometers 1, 2, and 3 for the GRF $Z$ axis. The differences are displayed before corrections (top row), after applying rotational cor- rections (middle row), after applying both rotational and gravity gradient corrections (bottom row), and after taking away the mean. The selected day is November 15, 2009

and in general higher drift values for the associated commonmode combinations.

The biases (direct) and drifts (indirect) of the individual accelerometers that are obtained by POD are thus internally consistent at a very high level. The question arises if these values are reliable in an absolute sense. A possibility is to validate these values by a comparison with differential biases that can be derived for the gravity gradients. In (Bouman et al. 2011), it is explained that the gravity gradients are not calibrated in an absolute sense: only the scale factors have been reliably determined for the measurement bandwidth. The gravity gradients suffer from low frequency noise resulting in among other drifting biases. These biases can be estimated by comparing the observed gravity gradients with those predicted by a gravity field model, in this case GOCO03s (GOCO 2014). When comparing the diagonal SGG components with those predicted by the GOCO03s gravity field model for daily arcs (again midnight to midnight), typically mean offsets are obtained that range up to 1000 Eötvös Units (EU or $1 \mathrm{~nm} / \mathrm{s}^{2} / \mathrm{m}$ or $10^{-9} \mathrm{~s}^{-2}$ ). These offsets $O$ should in principle be equal to 
Accelerometer
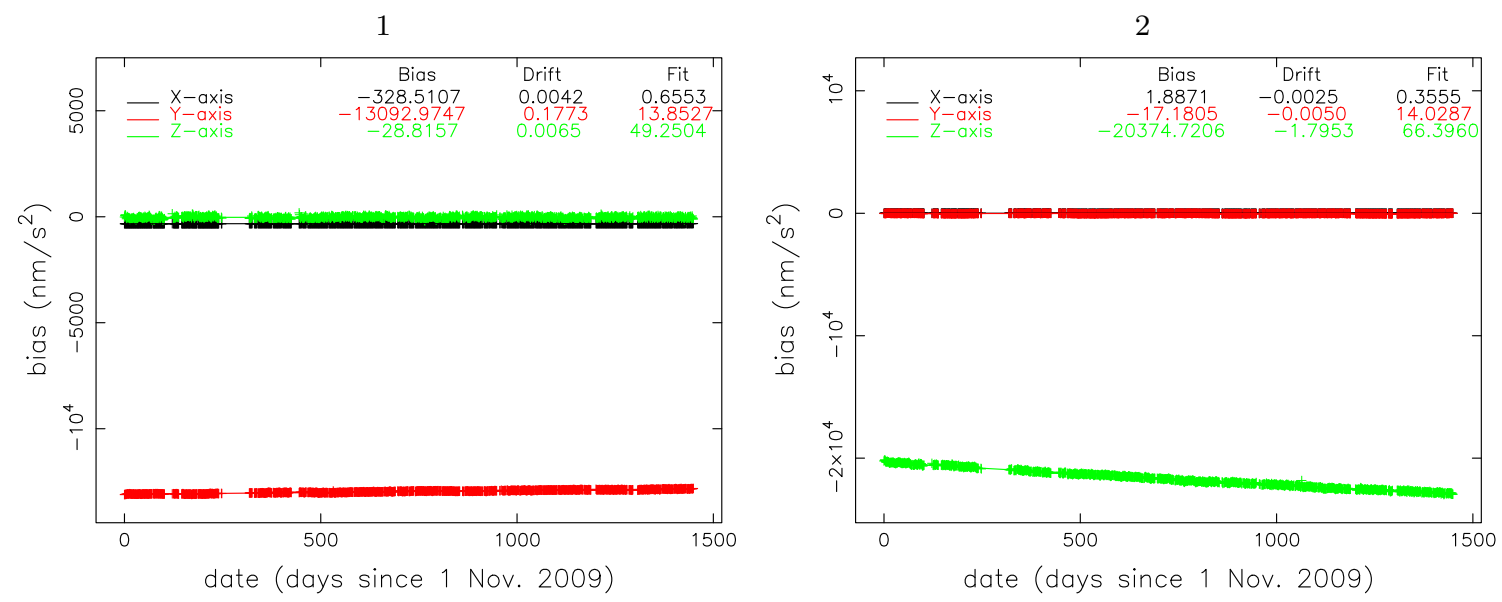

Accelerometer

3

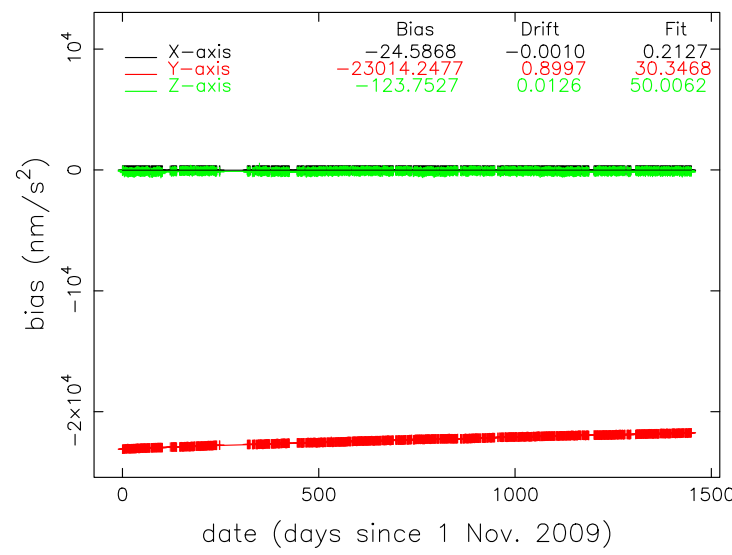

Fig. 6 Estimated accelerometer biases $\left(\mathrm{nm} / \mathrm{s}^{2}\right)$ and drifts $\left(\mathrm{nm} / \mathrm{s}^{2} /\right.$ day) for the GOCE accelerometers 1 to 4 for the $X, Y$, and $Z$ axes. Use was made of 1213 daily arcs. The RMS-of-fit for

$O\left(\Gamma_{x x}\right)=\left(b_{x, 1}-b_{x, 4}\right) / a_{x}$

$O\left(\Gamma_{y y}\right)=\left(b_{y, 2}-b_{y, 5}\right) / a_{y}$

$O\left(\Gamma_{z z}\right)=\left(b_{z, 3}-b_{z, 6}\right) / a_{z}$,

where $\Gamma_{i i}$ represent the diagonal gravity gradient components, with the index $i$ denoting the GRF axis $(i=x, y, z)$. The arm lengths of the orthogonal accelerometer pairs are indicated by $a_{i}$, and the daily individual accelerometer biases and drifts by $b_{i, j}$, where the index $j$ specifies the accelerometer $(j=1, \ldots, 6)$. These equations lead to the values as displayed in Fig. 8. A total of 1160 days with gravity gradient observations could be identified without outliers (e.g., due to satellite shaking for calibration purposes, see Table 1 in Visser et al. 2014) out of the 1213 days used for the estimation of individual accelerometer biases. The consistency between daily gradiometer biases from the individual accelerometer bias estimates and from the comparison

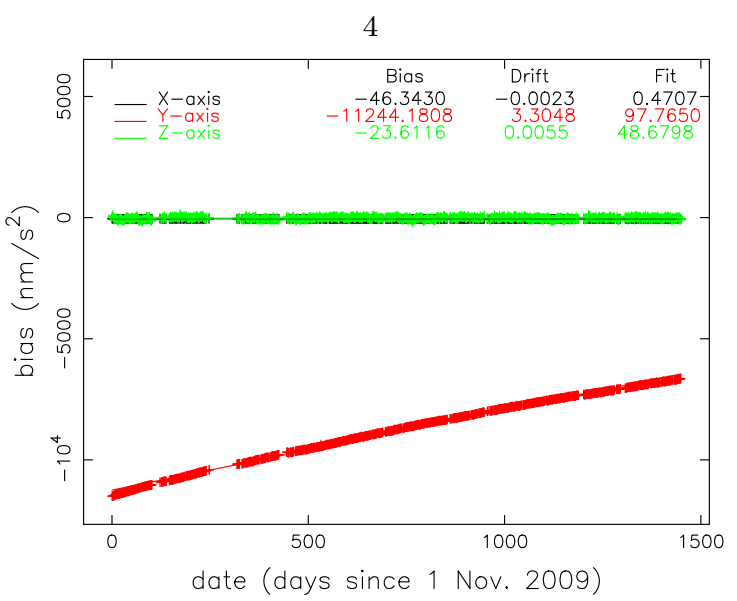

a linear regression is displayed as well $\left(\mathrm{nm} / \mathrm{s}^{2}\right)$, together with the bias (epoch November 1, 2009) and drift of the linear regression

between observed and modeled gravity gradients is -0.01 , 2.00 , and $-1.63 \mathrm{EU}$ in terms of mean and 0.02, 0.79, and $27.29 \mathrm{EU}$ in terms of rms-about-mean for the $X, Y$, and $Z$ axes, respectively (after applying 5- $\sigma$ editing leaving 1113 daily values out of the 1160 available common days). This level of consistency is in agreement with the level of consistency between the bias estimates of the individual accelerometers.

When scrutinizing Figs. 6, 7, and 8, it seems like some of the biases display an exponentially decaying trend, i.e., deviating from a pure linear drift. It is interesting to note that for the GRACE accelerometers, different second-order polynomials models are recommended to correct their observations (Bettadpur 2009), without giving a physical explanation for this apparent behavior of the accelerometers. The electronics of the GOCE accelerometers are subject to aging which contributes to their biases, but according to the manufacturer the anticipated contribution is much smaller than observed in 
Table 2 1D RMS-of-fit (cm) of PSO kinematic co-ordinates for daily arcs obtained by the dynamic orbit computation

\begin{tabular}{|c|c|c|c|c|c|c|c|}
\hline Acc. & $\mathrm{CM}^{1}$ & Acc. 1 & Acc. 2 & Acc. 3 & Acc. 4 & Acc. 5 & Acc. 6 \\
\hline $1-10$ November $2009^{1}$ & 5.18 & 5.20 & 14.78 & 9.99 & 5.24 & 14.89 & 8.42 \\
\hline $1-10$ November $2009^{2}$ & 5.18 & 5.27 & 7.15 & 9.95 & 5.43 & 6.84 & 8.41 \\
\hline $1-10$ November $2009^{3}$ & 5.18 & 5.17 & 5.18 & 5.21 & 5.23 & 5.17 & 5.25 \\
\hline Full mission ${ }^{3,4}$ & 6.87 & 6.89 & 6.87 & 6.92 & 6.92 & 6.97 & 6.92 \\
\hline
\end{tabular}

CM common-mode

${ }^{1}$ Uncorrected

${ }^{2}$ Corrected for rotational terms

${ }^{3}$ Fully corrected

${ }^{4}$ Full mission $=$ November 1, 2009-October 20, 2013
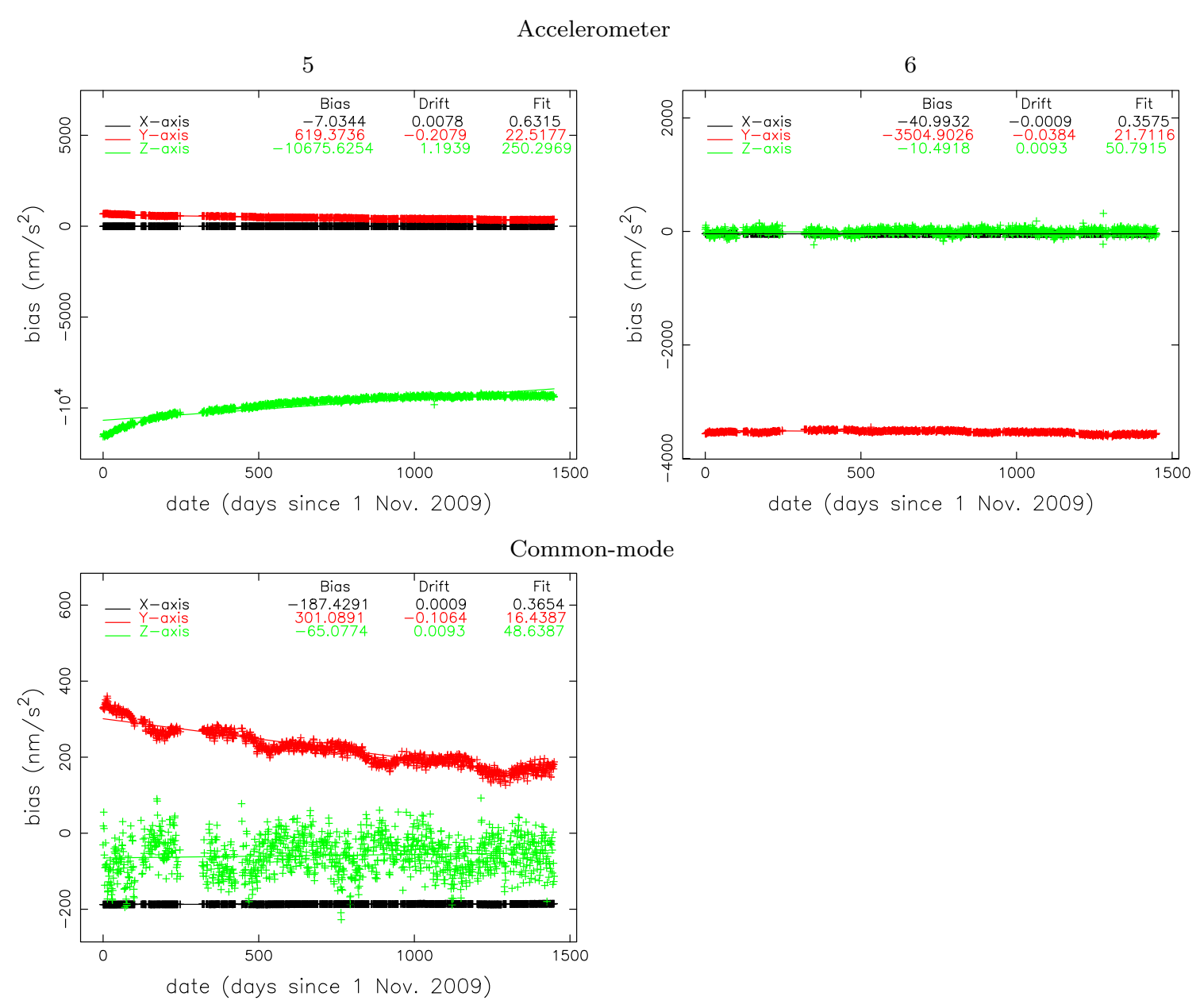

Fig. 7 Estimated accelerometer biases $\left(\mathrm{nm} / \mathrm{s}^{2}\right)$ and drifts $\left(\mathrm{nm} / \mathrm{s}^{2} /\right.$ day $)$ for the GOCE accelerometers 5 and 6 for the $X, Y$, and $Z$ axes. Use was made of 1213 daily arcs. The biases estimated for the common-mode

accelerometer observations are included for reference. The RMS-of-fit for a linear regression is displayed as well $\left(\mathrm{nm} / \mathrm{s}^{2}\right)$, together with the bias (epoch November 1, 2009) and drift of the linear regression

Figs. 6 and 7. Another possible contribution might come from a changing parasitic acceleration from the gold wire that connects the accelerometers with their cagings. In that case, the stiffness of this gold wire should change with time as well, which, however, is not anticipated either (Christophe 2015). For GOCE, two empirical functional models have been tested for describing the evolution of the observed accelerometer and gravity gradient biases (which are thus combinations of

biases of the associated individual accelerometers). These functional models are thus purely empirical and not based on a proved expected physical behavior of the accelerometers. The first model assumes a constant trend $d$ with $b_{0}$ the bias at epoch:

$b=b_{0}+d \times t$, 
Table 3 Comparison between accelerometer bias and drift values obtained by precise orbit determination using the fully corrected individual or common-mode $(\mathrm{CM})$ accelerometer observations

\begin{tabular}{|c|c|c|c|c|c|c|}
\hline & \multicolumn{3}{|l|}{$\operatorname{Bias}\left(\mathrm{nm} / \mathrm{s}^{2}\right)$} & \multicolumn{3}{|c|}{ Drift (nm/s²/day) } \\
\hline & $X$-axis & $Y$-axis & $Z$-axis & $X$-axis & $Y$-axis & $Z$-axis \\
\hline Acc. 1 & -328.5107 & $-13,092.9747$ & -28.8157 & 0.0042 & 0.1773 & 0.0065 \\
\hline Acc. 2 & 1.8871 & -17.1805 & $-20,374.7206$ & -0.0025 & -0.0050 & -1.7953 \\
\hline Acc. 3 & -24.5868 & $-23,014.2477$ & -123.7527 & -0.0010 & 0.8997 & 0.0126 \\
\hline Acc. 4 & -46.3430 & $-11,244.1808$ & -23.6116 & -0.0023 & 3.3048 & 0.0055 \\
\hline Acc. 5 & -7.0344 & 619.3736 & $-10,675.6254$ & 0.0078 & -0.2079 & 1.1939 \\
\hline Acc. 6 & -40.9932 & -3504.9026 & -10.4918 & -0.0009 & -0.0384 & 0.0093 \\
\hline Average & -187.4268 & 301.0966 & -67.1223 & 0.0010 & -0.1065 & 0.0110 \\
\hline $\mathrm{CM}$ & -187.4291 & 301.0891 & -65.0774 & 0.0009 & -0.1064 & 0.0093 \\
\hline
\end{tabular}

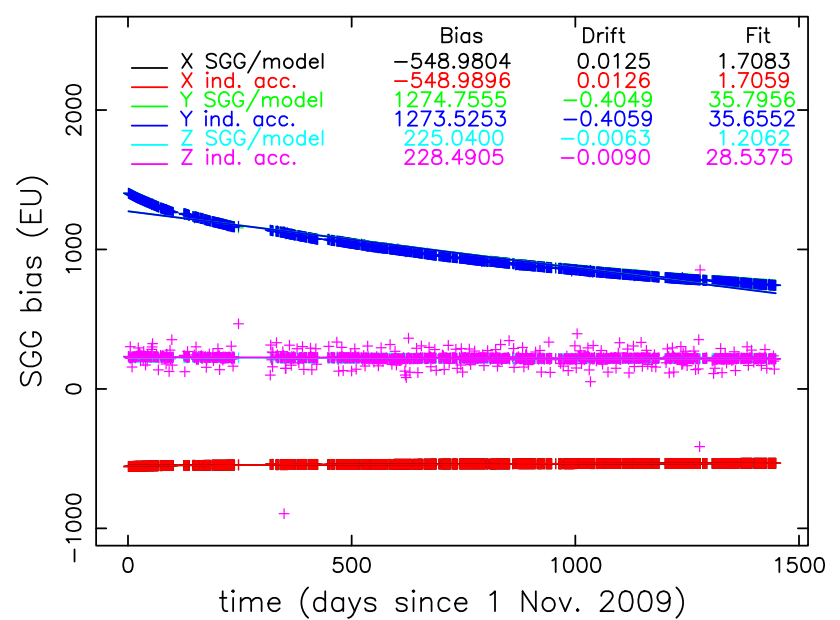

Fig. 8 Gradiometer biases derived from bias estimates of individual accelerometers and derived from a comparison between observed and modeled gravity gradients (Bias, Exponential scale factor, and RMSof-fit in EU, Drift in EU/day: values hold for 5- $\sigma$ editing). Please note that the outliers are included

where $t$ represents time since the epoch November 1, 2009 in days. The second one assumes an exponential decay with scale factor $s_{f}$ and decay time factor $t_{s}$ :

$b=b_{0}+s_{f} \times e^{-\frac{t}{t_{s}}}$

with $b$ representing either an accelerometer or gravity gradient bias. The associated parameters are again estimated by a least-squares regression, where for $t_{s}$ optimal values were obtained by trial and error. It was found that the second functional model hardly improved the RMS-of-fit for most accelerometer axes, with the exception of the $Y$ and $Z$ axes for accelerometer 5: the improvement is from 22.52 to $15.35 \mathrm{~nm} / \mathrm{s}^{2}$ and from 250.30 to $64.75 \mathrm{~nm} / \mathrm{s}^{2}$ for the $Y$ and $Z$ axes, respectively (Table 4 ). Accelerometer 5 thus appears to display a deviating behavior. The gravity gradient term $\Gamma_{y y}$ relies predominantly on the $Y$ axis of accelerometers 2 and 5. It can be observed that the second functional model significantly better describes the time evolution of the asso- ciated gravity gradient bias as well: the RMS-of-fit improves from 35.66 to $8.47 \mathrm{EU}$. Please note that for both the $Y$ axis of accelerometer 5 and for the $\Gamma_{y y}$ gravity gradient term, an optimal value of about 1000.0 days for $t_{s}$ was found. The respective scale factors $s_{f}$ are equal to about $408 \mathrm{~nm} / \mathrm{s}^{2}$ and 798 EU. Dividing the $Y$ axis scale factor $s_{f}$ for accelerometer 5 by the gradiometer arm length of $0.5 \mathrm{~m}$ leads to $816 \mathrm{EU}$. The difference of $18 \mathrm{EU}$ can be explained completely by the estimated scale factor $s_{f}$ for the $Y$ axis of accelerometer 2 which was found to be orders of magnitude smaller, namely equal to about $9 \mathrm{~nm} / \mathrm{s}^{2}$. A similar consistency is found for all the bias values $b_{0}$.

\subsection{Accelerometer scale factors}

The DFC system leaves a very small signal in the $X$ axis of typically less than $2 \mathrm{~nm} / \mathrm{s}^{2}$, which makes it as good as impossible to estimate accelerometer scale factors for this direction (Visser 2009). As stated before, the scale factors were already determined very reliably by other techniques (Bouman et al. 2011). Despite this, it is interesting and also possible to obtain reasonable estimates for the scale factors of the individual accelerometers. As also stated above, occasionally, the DFC is offset by a bias up to several hundreds to thousands of $\mathrm{nm} / \mathrm{s}^{2}$ in the $X$ direction for orbit correction, thus leaving a large non-gravitational signal that can be calibrated by POD. Therefore, for each accelerometer and for the common-mode accelerometer observations, sets of scale factors for the $X, Y$, and $Z$ directions were estimated on a yearly basis and for the full mission period in addition to the daily bias estimates by stacking the daily normal equations for the scale factor part (Helleputte and Van 2011). Please note that the estimated accelerometer scale factors are correlated with the estimated accelerometer biases. The impact of a scale factor deviating from 1 is, however, straightforward. Suppose the scale factor for a certain axis takes a value of $(1+\alpha)$, then the associated bias needs to be multiplied with $(1+\alpha)$ as well. In case only one set of scale factors is estimated for a certain period (e.g. on a yearly basis or for the full 
Table 4 Selected bias, drift, and scale factor parameters for the two functional models

\begin{tabular}{lllll}
\hline Equation 7 & Bias $b_{0}$ & Drift $d$ & RMS-of-fit & \\
\hline Acc. $5 Y$ & $619.37\left(\mathrm{~nm} / \mathrm{s}^{2}\right)$ & $-0.2079\left(\mathrm{~nm} / \mathrm{s}^{2} /\right.$ day $)$ & $22.52\left(\mathrm{~nm} / \mathrm{s}^{2}\right)$ & \\
Acc. $5 Z$ & $-10,675.63\left(\mathrm{~nm} / \mathrm{s}^{2}\right)$ & $\begin{array}{l}1.1939\left(\mathrm{~nm} / \mathrm{s}^{2} / \text { day }\right) \\
\Gamma_{y y}\end{array}$ & $\begin{array}{l}250.30\left(\mathrm{~nm} / \mathrm{s}^{2}\right) \\
35.66(\mathrm{EU})\end{array}$ & \\
\hline Equation 8 & Bias $b_{0}$ & Scale $s_{f}$ & RMS-of-fit & Time scale $t_{s}$ \\
\hline Acc. $5 Y$ & $253.36\left(\mathrm{~nm} / \mathrm{s}^{2}\right)$ & $407.9261\left(\mathrm{~nm} / \mathrm{s}^{2}\right)$ & $15.35\left(\mathrm{~nm} / \mathrm{s}^{2}\right)$ & $1000.0($ days $)$ \\
Acc. $5 Z$ & $-9262.56\left(\mathrm{~nm} / \mathrm{s}^{2}\right)$ & $-2149.0261\left(\mathrm{~nm} / \mathrm{s}^{2}\right)$ & $64.75\left(\mathrm{~nm} / \mathrm{s}^{2}\right)$ & $375.0($ days $)$ \\
$\Gamma_{y y}$ & $557.82(\mathrm{EU})$ & $798.4522(\mathrm{EU})$ & $8.63(\mathrm{EU})$ & 1000.0 (days) \\
\hline
\end{tabular}

The values for the RMS-of-fit of the respective regressions are included as well mission period: see below), the time patterns of the daily bias estimates thus stay the same, apart from a constant factor $\alpha$.

The estimated scale factors are displayed in Table 5. For the $X$ axis, the scale factors are very close to 1 for each year (also for the only 2 months of data available for 2009) and for the full mission period: the deviation from 1 is always smaller than $10^{-3}$ for both the common-mode and individual accelerometer observations. For the $Z$ axis, the scale factor varies between 0.968 and 1.002 and deviates less than $10^{-2}$ from 1 when using the full mission period. For the $Y$ axis, however, a persistent deviation is found of about 0.03 for all years and for the full mission period. The scale factors for the $Y$ axis suggest a systematic modeling deficiency in either the accelerometers themselves, the dynamic model or the kinematic orbit solutions. However, the same deviation is found for each accelerometer, which is an indication that these deviations are not caused by deficiencies in the accelerometers. Continuing efforts are made to improve tide models and mass change models for the atmosphere and oceans. It will be interesting to assess the sensitivity of the scale factor estimates to such improved models in the future. Perhaps, systematic errors in the kinematic orbit solutions cause the deviating
Table 5 Scale factors estimated by dynamically fitting the PSO kinematic co-ordinates for the selected 1213 daily arcs

\begin{tabular}{|c|c|c|c|c|c|c|c|}
\hline & $\mathrm{CM}$ & 1 & 2 & 3 & 4 & 5 & 6 \\
\hline \multicolumn{8}{|c|}{ 2009, 62 daily arcs } \\
\hline$X$ axis & 1.00009 & 1.00011 & 1.00015 & 1.00007 & 1.00009 & 1.00013 & 0.99998 \\
\hline$Y$ axis & 1.02669 & 1.02586 & 1.02724 & 1.02893 & 1.03036 & 1.02789 & 1.02904 \\
\hline$Z$ axis & 0.97686 & 0.97842 & 0.98030 & 0.97435 & 0.97276 & 0.97199 & 0.97778 \\
\hline \multicolumn{8}{|c|}{ 2010, 243 daily arcs } \\
\hline$X$ axis & 1.00012 & 1.00017 & 1.00019 & 0.99999 & 1.00009 & 1.00015 & 1.00000 \\
\hline$Y$ axis & 1.02653 & 1.02700 & 1.02596 & 1.02679 & 1.02765 & 1.02711 & 1.02718 \\
\hline$Z$ axis & 0.99365 & 0.99163 & 0.98475 & 0.97882 & 0.97868 & 0.99375 & 0.98197 \\
\hline \multicolumn{8}{|c|}{2011,323 daily arcs } \\
\hline$X$ axis & 0.99997 & 1.00003 & 1.00006 & 0.99980 & 0.99992 & 1.00001 & 0.99985 \\
\hline$Y$ axis & 1.03619 & 1.03781 & 1.03595 & 1.03704 & 1.03495 & 1.03688 & 1.03743 \\
\hline$Z$ axis & 0.97388 & 0.97606 & 0.97616 & 0.97751 & 0.96843 & 0.96982 & 0.97547 \\
\hline \multicolumn{8}{|c|}{2012,328 daily arcs } \\
\hline$X$ axis & 0.99987 & 0.99979 & 0.99994 & 1.00005 & 0.99996 & 0.99999 & 0.99988 \\
\hline$Y$ axis & 1.03567 & 1.03842 & 1.03501 & 1.03713 & 1.03476 & 1.03725 & 1.03617 \\
\hline$Z$ axis & 0.98430 & 0.98497 & 0.98602 & 0.98878 & 0.98125 & 0.98131 & 0.98799 \\
\hline \multicolumn{8}{|c|}{ 2013, 257 daily arcs } \\
\hline$X$ axis & 0.99994 & 0.99997 & 1.00003 & 0.99986 & 0.99993 & 1.00000 & 0.99986 \\
\hline$Y$ axis & 1.02796 & 1.02942 & 1.02751 & 1.02873 & 1.02743 & 1.02845 & 1.02759 \\
\hline$Z$ axis & 1.00110 & 1.00137 & 1.00154 & 1.00159 & 1.00010 & 1.00053 & 1.00081 \\
\hline \multicolumn{8}{|c|}{ Full mission period: 1 November 2009-20 October 2013, 1213 daily arcs } \\
\hline$X$ axis & 0.99994 & 0.99992 & 1.00001 & 0.99997 & 0.99997 & 1.00002 & 0.99989 \\
\hline$Y$ axis & 1.03087 & 1.03225 & 1.03048 & 1.03186 & 1.03078 & 1.03171 & 1.03141 \\
\hline$Z$ axis & 0.99557 & 0.99590 & 0.99603 & 0.99612 & 0.99323 & 0.99438 & 0.99557 \\
\hline
\end{tabular}


cross-track scale factor values. It is, for example, well known that kinematic orbit solutions are especially sensitive in the cross-track direction for phase center variation corrections (Bock et al. 2011). However, it is shown in Bock et al. (2011) that the kinematic orbit solutions have a very high level of precision, also in the cross-track direction, validated by independent Satellite Laser Ranging (SLR) observations.

\section{Summary and conclusions}

A methodology that was developed before the launch of GOCE for estimating biases of all individual accelerometers was successfully implemented and applied to the real accelerometer observations for the full mission period (amounting to 1213 usable daily arcs). When taking the rotational and gravity gradient corrections into account, the consistency between the observations taken by the individual accelerometers ranges between 0.02 and $0.2 \mathrm{~nm} / \mathrm{s}^{2}$ for the sensitive axes, and up to $1.0 \mathrm{~nm} / \mathrm{s}^{2}$ for the less sensitive axes in terms of RMS-about-mean. Without these corrections, the RMS-about-mean of differences between individual accelerometer observations ranges up to $40 \mathrm{~nm} / \mathrm{s}^{2}$.

It was found that rotational and gravity gradient corrections as applied to the individual accelerometers are required in order to have consistent orbital fits, comparable to the ones obtained with the common-mode accelerometer observations. The 1-dimensional RMS-of-fit is of the order of $7 \mathrm{~cm}$ for the entire GOCE operational mission period. Without applying the rotational and gravity gradient corrections, this fit can be up to $15 \mathrm{~cm}$. In addition, by this methodology, accelerometer bias estimates and drifts were obtained that are not only internally consistent, but also consistent with the biases that are present in the GOCE SGG observations.

For the $X$ and $Y$ axes (predominantly along-track and cross-track), a consistency between bias estimates for the common-mode accelerometer observations on the one hand and individual accelerometers observations on the other hand of within 0.01 and $0.0001 \mathrm{~nm} / \mathrm{s}^{2} /$ day was obtained for the bias and drift. For the $Z$-axis, the consistency is about 2 and $0.02 \mathrm{~nm} / \mathrm{s}^{2} /$ day, respectively.

The consistency between daily gradiometer biases from the individual accelerometer bias estimates and from the comparison between observed and modeled gravity gradients is $-0.01,2.00$, and $-1.63 \mathrm{EU}$ in terms of mean and $0.02,0.79$, and $27.29 \mathrm{EU}$ in terms of rms-about-mean for the $X, Y$ and $Z$ axes, respectively. This level of consistency is in agreement with the level of consistency between the bias estimates of the individual accelerometers.

Finally, because of occasional maneuvering, it was possible to estimate scale factors for the accelerometers by POD as well. The correctness of the gravity gradient scale factors is confirmed to within $1 \times 10^{-4}$ for the $X$ axis and $5 \times 10^{-3}$ for the $Z$ axis. For the $Y$ axis, a deviation of about 0.03 was obtained, which is small, but the same deviation was found for all accelerometers. The latter leaves an interesting question for future research, namely improvement of the dynamic force modeling for GOCE especially for the crosstrack direction.

Acknowledgments The European Space Agency is acknowledged for supporting this study and providing the GOCE observations. Part of the computations relied on the GEODYN software that was kindly provided by the NASA Goddard Space Flight Center, Greenbelt, Maryland.

Open Access This article is distributed under the terms of the Creative Commons Attribution 4.0 International License (http://creativecomm ons.org/licenses/by/4.0/), which permits unrestricted use, distribution, and reproduction in any medium, provided you give appropriate credit to the original author(s) and the source, provide a link to the Creative Commons license, and indicate if changes were made.

\section{References}

Bettadpur S (2009) Recommendation for a-priori bias \& scale parameters for level-1B ACC data (Version 2), GRACE TN-02. Version 2:17

Bock H, Jäggi A, Meyer U, Visser P, van den IJssel J, Van Helleputte T, Heinze M, Hugentobler U (2011) GPS derived orbits for the GOCE satellite. J Geod 85:807-818. doi:10.1007/s00190-011-0484-9

Bock H, Jäggi A, Meyer U, Dach R, Beutler G (2011) Impact of GPS antenna phase center variations on precise orbits of the GOCE satellite. Adv Space Res 47(11):1885-1893. doi:10.1016/j.asr. 2011.01.017

Bock H, Jäggi A, Beutler G, Meyer U (2014) GOCE: precise orbit determination for the entire mission. J Geod 88(11):1047-1060. doi:10.1007/s00190-014-0742-8

Bouman J, Fiorot S, Fuchs M, Gruber T, Schrama E, Tscherning C, Veicherts M, Visser P (2011) GOCE gravitational gradients along the orbit. J Geod 85:791-805. doi:10.1007/s00190-011-0464-0

Cesare C, Catastini G (2005) Gradiometer on-orbit calibration procedure analysis, Technical Note to ESA, GO-TN-AI-0069, Issue 3, Alenia Aerospazio

Christophe B (2015) ONERA, France priv comm

Drinkwater M, Haagmans R, Muzzi D, Popescu A, Floberghagen R, Kern M, Fehringer M (2007), The GOCE gravity mission: ESA's first core explorer, In: $3^{\text {rd }}$ GOCE user workshop, 6-8 November 2006, Frascati, Italy, pp. 1-7, ESA SP-627

ESA (1999) Gravity field and steady-state Ocean Circulation Mission. Reports for mission selection, the four candidate Earth Explorer Core Missions. SP-1233(1)

Frommknecht B, Lamarre D, Meloni M, Bigazzi A, Flobreghagen R (2011) GOCE level 1b data processing. J Geod 85:759-775. doi:10.1007/s00190-011-0497-4

GOCO (2014) Gravity observation combination (GOCO) www.goco. eu. Accessed 9 May 2014

Gruber T, Abrikosov O, and Hugentobler O (2010) GOCE standards. Doc No: GO-TN-HPF-GS-0111, Issue 3 Rev. 2

Helleputte TV (2011) The integration of spaceborne accelerometry in the precise orbit determination of low-flying satellites. Ph.D. Dissertation, Delft University of Technology, Delft, January 2011, Printed by: DC: Print \& Sign, Zelzate

McCarthy DD and Petit G (2004) IERS conventions (2003) IERS Technical Note 32, Frankfurt am Main: Verlag des Bundesamts für Kartographie und Geodäsie, 127 pp. paperback, ISBN 3-89888884-3 (print version) 
Montenbruck O, Gill E (2000) Satellite orbits—models methods applications. Springer, Berlin ISBN 3-540-67280-X

Pail R, Bruinsma S, Migliaccio F et al (2011) First GOCE gravity field models derived by three different approaches. J Geod 85:819-843. doi:10.1007/s00190-011-0467-x

Pavlis DE, Poulouse S, McCarthy JJ (2006) GEODYN operations manual, contractor report. SGT Inc., Greenbelt

Ray RD (1999) A global ocean tide model from topex/poseidon altimetry: GOT99.2, NASA Tech Memo Goddard Space Flight Center 209478 p 58

Rispens S, Bouman J (2009) Calibrating the GOCE accelerations with star sensor data and a global gravity field model. J Geod 83:737749. doi:10.1007/s00190-008-0290-1

Siemes C, Haagmans R, Kern M, Plank G, Floberghagen R (2012) Monitoring GOCE gradiometer calibration parameters using accelerometer and star sensor data: methodology and first results. J Geod 86:629-645. doi:10.1007/s00190-012-0545-8
Standish EM (1998) JPL planetary and lunar ephemerides, DE405/LE405, JPL IOM 312.F-98-048

Visser PNAM (2008) Exploring the possibilities for star-tracker-assisted validation of the six individual GOCE accelerometers. J Geod 82(10):591-600. doi:10.1007/s00190-007-0205-6

Visser PNAM (2009) GOCE gradiometer: estimation of biases and scale factors of all six individual accelerometers by precise orbit determination. J Geod 83(1):69-85. doi:10.1007/s00190-008-0235-8

Visser PNAM, van der Wal W, Schrama EJO, van den IJssel J, Bouman J (2014) Assessment of observing time-variable gravity from GOCE GPS and accelerometer observations. J Geod 88(11):1029-1046. doi:10.1007/s00190-014-0741-9 